\title{
Advances in evaluating the fetal skeleton
}

This article was published in the following Dove Press journal:

International Journal of Women's Health

13 May 2014

Number of times this article has been viewed

\author{
Ann-Edwidge Noel \\ Richard N Brown \\ Division of Maternal Fetal Medicine, \\ Department of Obstetrics and \\ Gynecology, McGill University, \\ Montreal, QC, Canada
}

\begin{abstract}
In this review, we discuss aspects of the prenatal diagnosis of fetal skeletal malformations, concentrating on the advantages offered by different imaging techniques and the approaches that are of value in evaluating a suspected skeletal dysplasia. We also briefly address the findings in some of the commoner malformations of the fetal skeleton that may be encountered. Keywords: prenatal diagnosis, skeletal dysplasia, ultrasound, fetal imaging, osteodysplasia, chondrodysplasia, dysostosis
\end{abstract}

\section{Introduction}

Congenital skeletal diseases are well described, but nevertheless continue to present significant diagnostic challenges. Skeletal abnormalities comprise a diverse and complex group of disorders that impact upon the growth and development of both bone and cartilage, resulting in abnormalities represented by variations in the morphology of different segments of the skeleton. Their etiologies may be broadly categorized as having intrinsic or extrinsic causes, the former including characteristics seen as part of chromosomal abnormalities or those single-gene disorders where there is involvement of imprinting errors of metabolism, while the latter group comprises, eg, the consequences of teratogen exposure (eg, thalidomide, a cause of phocomelia and proximal femoral focal deficiency) or of autoimmune processes.

Historically, these disorders were described using the terms osteodysplasia, chondrodysplasia and dysostosis. Osteodysplasias are generalized skeletal malformations and chondrodysplasias represent anomalies of cartilage development, while the dysostoses are disorders affecting individual or specific groups of bones. In clinical practice, the distinction between these three entities has become blurred, and typically the various abnormalities are now usually referred to more broadly as skeletal dysplasias.

The subclassification of skeletal dysplasias has been revised several times over the last four decades; in the latest revision published in 2010, some 456 conditions are included in the International Nosology and Classification of Genetic Skeletal Disorders. These have been subdivided into 40 categories defined by a combination of molecular, biochemical, and radiographic criteria. Three hundred and sixteen of these are associated with mutations in one or more of 226 individual genes, and thus molecular genetics has come to play a significant role in the diagnosis of skeletal dysplasias. ${ }^{1}$

Osteo- and chondrodysplasias are evident following the embryonic period, and typically have a phenotypic expression that continues to evolve throughout life. In contrast, the dysostoses, which represent errors in blastogenesis, are skeletal 
malformations that arise during the embryogenic period and have a phenotype that remains stable.

Although many of the individual disorders comprising skeletal dysplasias and dysostoses are rare, the overall birth prevalence of skeletal dysplasias is estimated to be 2.4-4 of 10,000 births. $^{2}$ The prevalence during pregnancy appears to be greater, at 7.5 of 10,000 pregnancies, ${ }^{3}$ and these disorders are identified in $0.9 \%$ of perinatal deaths.

Since the skeleton develops early in pregnancy, prenatal diagnosis of skeletal dysplasias is possible, with their identification and evaluation by ultrasound being the primary investigative tool. Ideally, the role of prenatal skeletal imaging is to establish a clear differential diagnosis as early as possible, so that further investigations, such as molecular diagnostic testing, can be considered where applicable, and to support the attainment of a more precise diagnosis, ideally before viability is achieved. This will permit informed discussions surrounding the prognosis to be held with the parents and caregivers, and allow a management plan to be drawn up. This is best achieved through a multidisciplinary specialty approach, most typically involving maternal fetal medicine, prenatal genetics, neonatology, and pediatric orthopedics. The distinction between lethal and nonlethal dysplasias is crucial in counseling patients with respect to either termination of the pregnancy (where this is allowed) or continuation with the pregnancy. In ongoing pregnancies, a thorough pediatric evaluation at birth is essential to confirm the suspected diagnosis, but prenatal suspicion of lethality may facilitate both access to and parental acceptance of postnatal palliative care in those cases where lethality is confirmed.

The large variety and variability in the presentation of skeletal dysplasias and their widely overlapping features significantly complicates accurate diagnosis. This remains true in spite of advances in skeletal imaging, the use of magnetic resonance imaging (MRI) and other imaging tools to complement ultrasound and improvements in molecular and biochemical testing. For many conditions, there is as yet no specific molecular or genetic testing available even if invasive testing is undertaken. A further impediment to prenatal diagnosis arises because some skeletal dysplasias do not present during the second trimester and only become evident in the late prenatal period or even after birth, and therefore are not identified at the time that fetal anatomy is routinely assessed. Therefore postdelivery imaging and physical evaluation, as well as radiographic and autopsy examination in lethal disorders, are often relied upon to provide a definitive diagnosis.
In this review, we outline the different imaging modalities that can be useful in the diagnosis of these disorders, and although we in the main concentrate upon the evaluation of skeletal dysplasias, we also address some other specific skeletal malformations.

\section{Roles of different imaging modalities in skeletal examination Ultrasound}

Ultrasound examination of the fetus offers us the opportunity to identify a variety of skeletal and bony abnormalities within the fetus and allows for the differentiation of various severe and often lethal skeletal dysplasias. However, in isolation it remains a poor tool at being able to discriminate between the various skeletal dysplasias with certainty. ${ }^{4}$

The skeleton begins to ossify early in development, and can therefore be assessed by ultrasound throughout most of pregnancy. Vertebral arch ossification is apparent by the eighth week, whilst cranial vault ossification begins around the ninth week. The clavicle, mandible, ileum, scapula, and long bones ossify by 12 weeks of gestation, and the metacarpals and metatarsals are ossified by $12-16$ weeks. The talus and calcaneus ossify between weeks 22 and 24, whereas the carpal bones ossify after birth. Epiphyses are delineated around 20 weeks.

\section{Two-dimensional ultrasound}

The prenatal identification of a skeletal dysplasia is most often prompted either by the incidental finding of an abnormally short femur or in light of a familial history of skeletal dysplasia. ${ }^{5}$ A detailed sonographic assessment is mandatory in all cases where a skeletal dysplasia or malformation is suspected. The detection of skeletal diseases of prenatal onset has improved enormously with advances in 2-D imaging. However, the sensitivity of 2-D ultrasound, which represents the current standard of care for prenatal diagnosis, remains limited, ranging most typically between $40 \%$ and $60 \%$. Antenatal sonography performs better when evaluating lethal skeletal dysplasias. Table 1 summarizes the diagnostic accuracy of 2-D ultrasound in the prenatal diagnosis of skeletal dysplasias, and as can be noted, there has been a clear improvement over time, due in part to improvements in imaging technology and also due to improvements in our understanding of these diverse conditions.

Kurtz et al first demonstrated the role that ultrasound offers in the diagnosis of these disorders in 1983, with a prospective study of 15 women and 16 cases. The diagnostic accuracy, however, was only $30 \% .{ }^{6}$ Parilla et al identified 
Table I Diagnostic accuracy of ultrasound in assessing skeletal dysplasias

\begin{tabular}{|c|c|c|c|}
\hline Study & Year & $\begin{array}{l}\text { Number } \\
\text { of cases }\end{array}$ & $\begin{array}{l}\text { Correct } \\
\text { diagnosis \% (n) }\end{array}$ \\
\hline Kurtz and Wapner 6 & 1983 & 16 & $31(5)$ \\
\hline Gaffney et $\mathrm{al}^{47}$ & 1998 & 35 & $31(11)$ \\
\hline Tretter et al ${ }^{48}$ & 1998 & 27 & $48(13)$ \\
\hline Hersh et $\mathrm{al}^{49}$ & 1998 & 23 & 48 (II) \\
\hline Doray et $\mathrm{al}^{50}$ & 2000 & 47 & $60(28)$ \\
\hline Parilla et $\mathrm{al}^{7}$ & 2003 & 31 & $65(20)$ \\
\hline Ruano et $\mathrm{al}^{23}$ & 2004 & 6 & $66(4)$ \\
\hline Krakow et $\mathrm{al}^{8}$ & 2008 & 405 & $78(3 \mid 6)$ \\
\hline Schramm et al $^{9}$ & 2009 & 162 & $68(110)$ \\
\hline
\end{tabular}

37 cases of skeletal dysplasias antenatally over an 8-year period. Complete follow-up was obtained in 31 cases, and the antenatal diagnosis was confirmed to be correct in $20(65 \%)$; these included thanatophoric dysplasia (eight), osteogenesis imperfecta (six), Roberts syndrome (two), achondroplasia (three), Ellis-van Creveld syndrome (one), metaphyseal dysplasia (one), spondyloepiphyseal dysplasia (one), distal arthrogryposis (one), caudal regression (one), and glycogenstorage disorder (one). There were two false-positive diagnoses, but the accuracy in predicting lethality was $100 \%$ with no false positives among these, although it must be borne in mind that this was a small series. The most common predictors of lethal skeletal dysplasias included early and severe shortening of the long bones ( first percentile), a femur length/abdominal circumference ratio of less than 0.16 , hypoplasia of the thorax, and certain other distinguishing characteristics, like marked bowing or fractures, short ribs, caudal regression, and cloverleaf skull. ${ }^{7}$

Krakow et al also conducted a retrospective analysis evaluating 1,500 cases referred to the International Skeletal Dysplasia Registry to determine the relative frequency of specific osteochondrodysplasias and the correlation between ultrasound and radiographic diagnoses; $85 \%$ of the referred cases were well-defined skeletal dysplasias, while the other $15 \%$ were a mixture of genetic syndromes and cases of probable early onset intrauterine growth restriction. The three commonest prenatal-onset skeletal dysplasias were osteogenesis imperfecta type II, thanatophoric dysplasia and achondrogenesis type II, accounting between them for almost $40 \%$ of the cases. The correlation between the referral ultrasound diagnosis and final radiographic diagnosis in these more commonly seen skeletal disorders was approximately $50 \%$. In a prospective analysis of 500 cases using a standardized ultrasound approach for the evaluation of skeletal disorders, the rates of recognition of osteogenesis imperfecta type II, thanatophoric dysplasia, and achondrogenesis type II were similar to those observed in the retrospective analysis. ${ }^{8}$ Schramm et al demonstrated the accuracy of prenatal ultrasound examination to be $68 \%$, with another $31 \%$ having a partially correct diagnosis and only $0.07 \%$ being false positives. ${ }^{9}$

Since the introduction of first-trimester ultrasound screening for Down syndrome, there has also been a growing interest in undertaking late first-trimester anatomical assessments. This has been aided both by improvements in 2-D ultrasound resolution and advances in and the use of transvaginal ultrasound for the assessment of fetal anatomy. From Down syndrome screening programs, it is clear that elevated nuchal translucency (NT) may also act as a marker for other fetal pathologies, and there is now substantial evidence that in fetuses with increased NT and a normal karyotype, the prevalence of skeletal dysplasias is increased. One possible explanation for the elevated NT in these cases might be through the mechanism of mediastinal compression due to a narrowed thorax, and in more extreme cases reduced fetal movements due to limb fractures; abnormalities of the skin composition and elasticity associated with disorders of collagen synthesis may also be contributory in some cases. ${ }^{10,11}$

\section{Three-dimensional ultrasound}

3-D ultrasound expands the technical horizons achieved by 2-D imaging, but does not replace it; rather, the two should be considered complementary techniques. ${ }^{12,13} 3$-D ultrasound allows the acquisition and storage of multiple 2-D planes, which with the use of increasingly sophisticated computer algorithms, allows this volumetric data set to be reconstructed and displayed in any plane. This can be performed either in real time or offline once the data set has been stored and the patient examination completed. Not only does 3-D allow imaging in planes that cannot conventionally be visualized by 2-D examination, but the technique allows for multiplanar views to be assessed, for surfaces of structures to be examined in rendered 3-D representations, and also for certain tissue types (eg, bone) to be extracted from the volumetric data set. Planar images are usually more useful than rendered images for the evaluation and diagnosis of the majority of fetal anomalies. ${ }^{14}$ The latter technique is particularly useful in skeletal examination, as the calcified bones can be isolated from the remaining fetal tissues to produce an ultrasoundderived "radiograph" of the skeleton that can be visualized in any orientation or plane. ${ }^{15,16} 3-\mathrm{D}$ reconstruction of the fetal bones is best performed using the maximum-intensity projection mode, a rendering algorithm that prioritizes the 
display of the highest grayscale levels contained within a region of interest selected by the operator. ${ }^{17}$

Recent data supports the use of 3-D ultrasound to improve the accuracy of standard 2-D imaging in the evaluation of skeletal abnormalities. This modality may better permit visualization of certain phenotypic features than 2-D ultrasound. 3-D offers improved sensitivity in evaluation of anomalies of the extremities, facial dysmorphisms (such as low-set or deformed ears, micrognathia, flattening of the facial profile associated with midface hypoplasia and cranial distortion due to craniosynostosis), scapular anomalies and abnormal calcification patterns. Table 2 summarizes the phenotypic features that are best visualized with 3-D ultrasound. ${ }^{18-20}$

Diagnoses of osteogenesis imperfecta, ${ }^{21}$ short-rib polydactyly syndrome, ${ }^{22}$ and Apert syndrome ${ }^{20}$ have all been described using 3-D ultrasound, although no characteristics

Table 2 Phenotypic findings in skeletal dysplasias that have improved visualization by 3-D ultrasound compared with 2-D ultrasound

\begin{tabular}{|c|c|}
\hline Skeletal c & $\begin{array}{l}\text { Phenotypic characteristics better } \\
\text { identified by } 3-D \text { US than by } 2-D \text { US in } \\
\text { published reports }\end{array}$ \\
\hline $\begin{array}{l}\text { Platylospondylic } \\
\text { lethal chondrodysplasia }{ }^{13}\end{array}$ & $\begin{array}{l}\text { Enhanced visualization of femoral and tibial } \\
\text { bowing (Figure 2); better characterization of } \\
\text { the facial soft tissues with surface rendering }\end{array}$ \\
\hline Campomelic dysplasia ${ }^{21}$ & $\begin{array}{l}\text { Micrognathia (Figure 3); flat face; hypoplastic } \\
\text { scapulae; bifid foot }\end{array}$ \\
\hline $\begin{array}{l}\text { Thanatophoric } \\
\text { dysplasia }^{21}\end{array}$ & $\begin{array}{l}\text { Improved characterization of frontal bossing } \\
\text { and depressed nasal bridge; demonstration of } \\
\text { redundant skin folds; low-set dysmorphic ears }\end{array}$ \\
\hline Achondroplasia ${ }^{51}$ & $\begin{array}{l}\text { Improved characterization of frontal bossing } \\
\text { and depressed nasal bridge; superior evaluation } \\
\text { of the epiphyses and metaphyses of the long } \\
\text { bones, with demonstration of a vertical } \\
\text { metaphyseal slope; caudal narrowing of the } \\
\text { interpedicular distance; clear visualization } \\
\text { of trident hand; better visualization of } \\
\text { disproportion between limb segments }\end{array}$ \\
\hline $\begin{array}{l}\text { Chondrodysplasia } \\
\text { punctata - rhizomelic } \\
\text { form }^{20}\end{array}$ & $\begin{array}{l}\text { Improved characterization of the Binder facies } \\
\text { (depressed nasal bridge, mid-face hypoplasia } \\
\text { [Figure 4], small nose with upturned alae); } \\
\text { identification of laryngeal stippling }\end{array}$ \\
\hline Achondrogenesis ${ }^{20}$ & $\begin{array}{l}\text { Panoramic demonstration of short neck and } \\
\text { severe shortening of all segments of the limbs }\end{array}$ \\
\hline $\begin{array}{l}\text { Jarcho-Levin } \\
\text { syndrome }\end{array}$ & $\begin{array}{l}\text { Vertebral defects with absence of ribs and } \\
\text { transverse process }\end{array}$ \\
\hline $\begin{array}{l}\text { Spondylocostal } \\
\text { dysostosis }\end{array}$ & Fan-like rib cage with rib fusion \\
\hline Larsen syndrome ${ }^{54}$ & $\begin{array}{l}\text { Genu recurvatum, midface hypoplasia } \\
\text { (Figure } 4 \text { ) and low-set ears }\end{array}$ \\
\hline Cleidocranial dysplasia $^{55}$ & $\begin{array}{l}\text { Widened cranial sutures, poor mineralization } \\
\text { of occipital bones, pseudarthrosis of the } \\
\text { clavicle }\end{array}$ \\
\hline Apert syndrome $e^{56}$ & Coronal craniosynostosis \\
\hline
\end{tabular}

that would not also be visualized by 2-D were observed. Ruano et al compared 2-D with other imaging modalities in six cases of skeletal dysplasia. Four of the six were correctly diagnosed by 2-D ultrasound, while all were diagnosed correctly with the additional use of a combination of 3-D ultrasound and computed tomography (CT). ${ }^{23}$

Obtaining detailed structural information regarding the fetal spine and thorax is a key component in the evaluation of the fetus with a skeletal dysplasia. Abnormalities of the rib cage and anomalies of thoracic development may indicate a risk for subsequent pulmonary hypoplasia, and associated neural tube defects will significantly impact prognoses. 3-D ultrasound may facilitate evaluation of skeletal malformations that affect the thorax and spine, most notably including scoliosis, hemivertebrae, butterfly vertebrae, and abnormalities of the thoracic cage. ${ }^{15,24}$

Multiplanar views of the fetal pelvis have been studied, particularly with respect to assessment of the iliac angle, which is thought to be abnormally wide in Down syndrome. Lee et al have shown that axial iliac angle measurements by multiplanar 3-D ultrasound are reproducible and can be used to examine fetuses considered at risk for having trisomy 21 . This technique may offer benefits over 2-D ultrasound by reducing the interobserver variability that is typically associated with iliac angle measurement. ${ }^{25}$

3-D ultrasound also aids in the detailed evaluation of the fetal extremities, although movement of the fetal limbs makes capturing usable volumetric data somewhat challenging. Multiplanar imaging (correlated with rendered images) allows the examiner to standardize the orientation of the hand, enabling examination of the relationships of distal forearm bones, metacarpals, fingers, and the thumb in the same plane, and rendered images allow examination of limb posturing (Figure 1). The same techniques are also used to assess the lower extremities. ${ }^{15}$

3-D images are captured by one of two approaches. Most 3 -D transducers are mechanical in nature, containing a conventional 2-D linear ultrasound array that is swept or tilted mechanically within the transducer head (mechanical sector probes). Combined with accurate positional information on the 2-D array's location at each time point, the multiple 2-D images are stored and recreated into a volumetric data set that can then be manipulated. Such 3-D-capable ultrasound systems can generally display multiplanar images in three orthogonal planes only. The advent of matrix-array probes has created new possibilities in 3-D imaging. Instead of having a linear array of crystals, as found in most 2-D and mechanical 3 -D probes, these probes consist of a 2-D matrix of crystals 


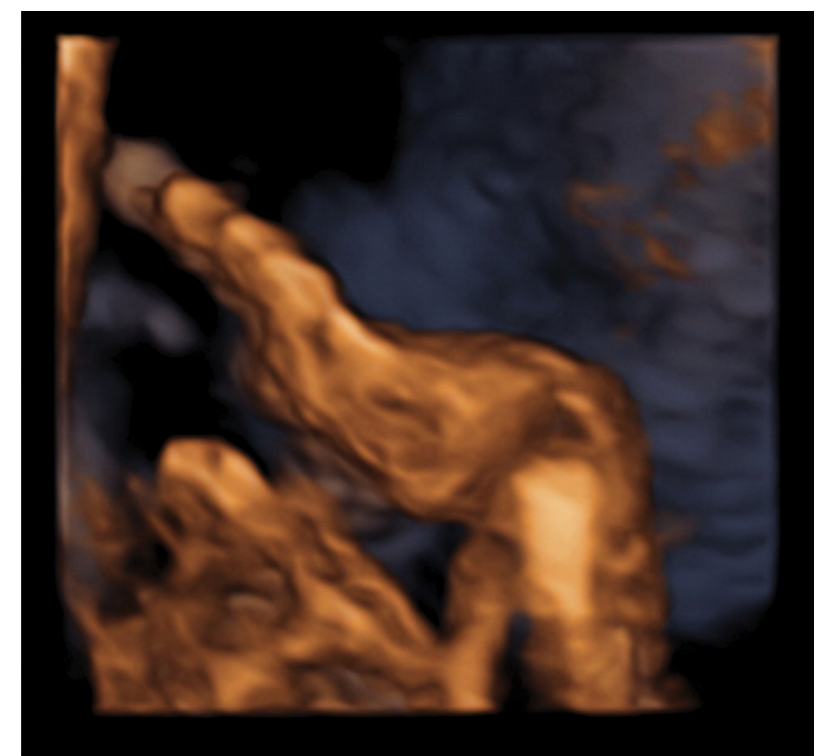

Figure I 3-D rendering of fetal hand, showing abnormal posturing due to metacarpal joint subluxation.

(often containing several thousand elements) that allow the ultrasound beam to be formed in any or in multiple planes in real time. Whereas planar views attained using mechanical probes can only be reconstructed after the data set has been captured, matrix probes allow this to be performed in real time during live imaging. This has led to the creation of a number of different imaging modalities, examples of which include 3-D extended imaging (3D XI; Samsung Medison, Seoul, Korea), an imaging-processing tool whose value in assessing fetal abnormalities has been studied by Leung et al. It incorporates the multislice-view and oblique-view modes. The multislice-view mode allows simultaneous display of multiple sequential parallel planes, while the oblique-view mode allows examination of a nonstandard plane that can be straight or curved. ${ }^{14}$ Coronal views along the curvature of the fetal spine might be of use in evaluating such conditions as spina bifida and hemivertebrae. The Live xPlane (Philips, Amsterdam, the Netherlands) allows simultaneous real-time visualization of two variable planes with detailed resolution.

3-D imaging systems do have limitations. 3-D techniques are generally limited by the same difficulties and restrictions that affect 2-D imaging and ultrasound in general, including unfavorable maternal body habitus, motion artifacts attributable to fetal or maternal movement, and decreased amniotic fluid, as is often associated with fetal malformations. ${ }^{15}$ More specifically with $3-\mathrm{D}$, it is important to appreciate that the quality of reconstructed multiplanar images not aligned to the original plane of

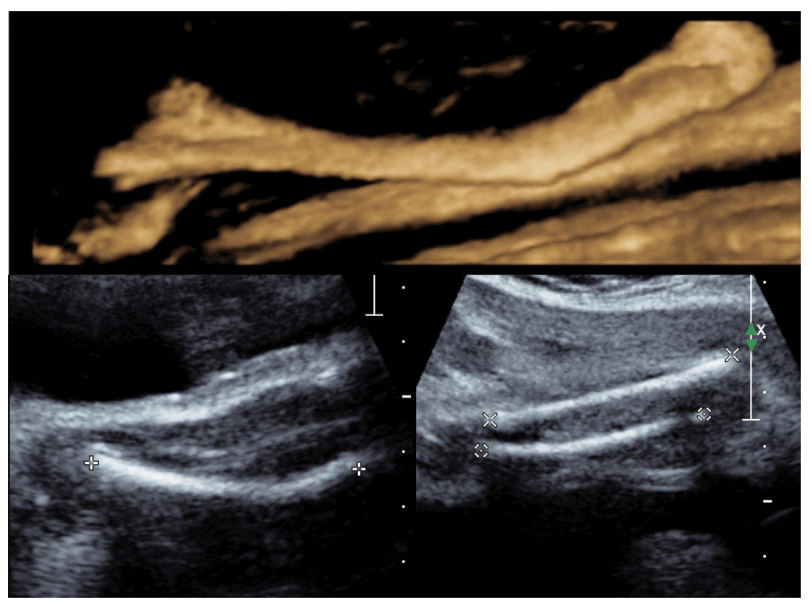

Figure 2 2-D image showing femoral angulation compared to straight distal long bones (tibia and fibula) with superimposed 3-D rendering of the femoral shaft.

acquisition is generally not as good in terms of detail or resolution as those obtained by conventional 2-D ultrasound, and that, as noted, most conventional orthogonal display modes do not allow the display of a sectional plane across a curved structure, such as the fetal spine, which may be especially useful in evaluating the skeleton. ${ }^{14}$

Although the availability and use of 3-D is increasingly widespread, there are at present insufficient data to validate the use of the 3-D modality alone as a diagnostic tool in the evaluation of skeletal malformations. Furthermore, it is essential to appreciate that the use of 3-D imaging relies upon the acquisition and interpretation of data represented in reconstructed 2-D planes. Rendered 3-D representations are complementary to this 2-D data, and can be useful in presenting image data to individuals not used to assessing ultrasound images, but the skills required to evaluate a 2-D image remain essential in using 3-D.

\section{Assessing skeletal dysplasias using 2-D and 3-D ultrasound}

Guidelines detailing the sonographic evaluation of the fetal skeleton have previously been presented. ${ }^{5,26-28}$ Table 3 summarizes the systematic approach that should be adopted when a fetal skeletal abnormality is suspected. Measurements of the femur and humerus are an essential component of any mid-trimester ultrasound evaluation, and often present the first clue to the presence of a skeletal dysplasia. Appropriate measurements extend along the ossified portion of the femur from the major trochanter to the distal end of the femoral shaft, with the proximal and distal epiphyses not being included; if necessary, several measurements should be taken to ensure accurate evaluation. 


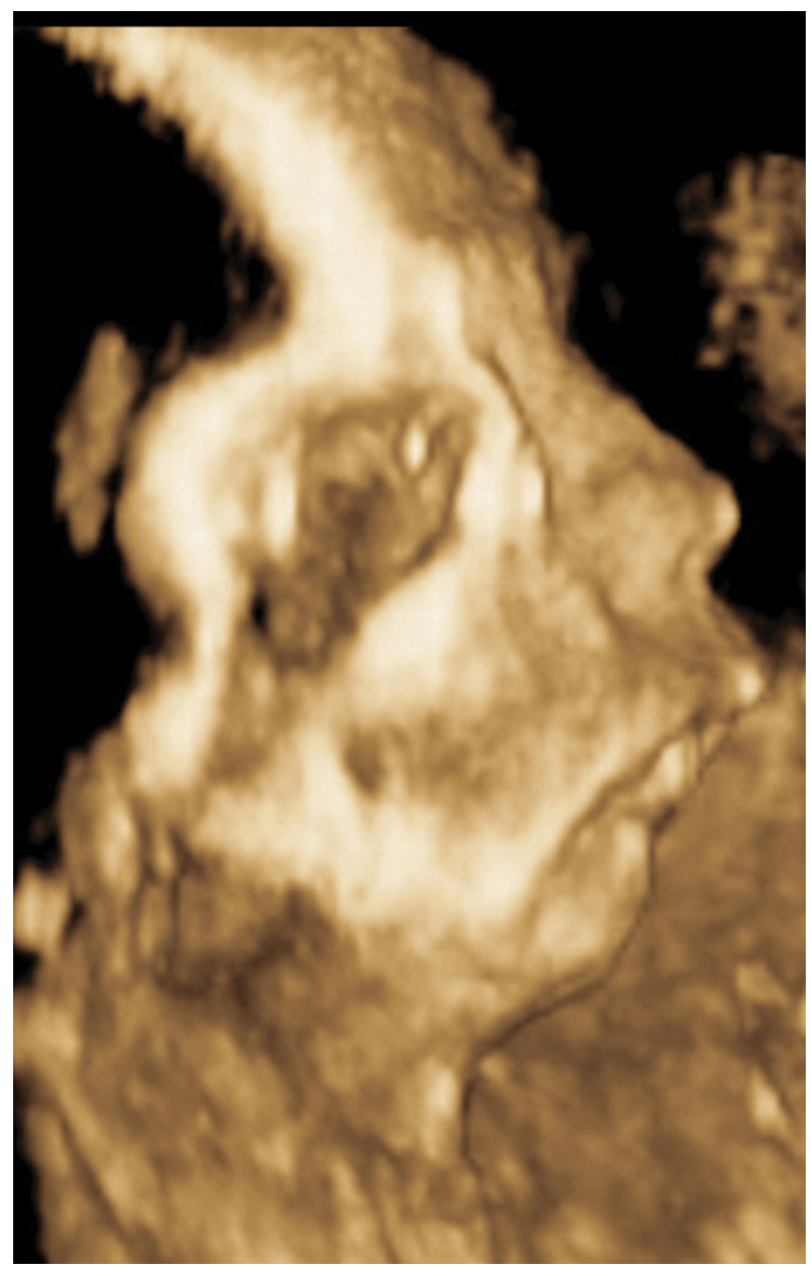

Figure 3 3-D rendering of the fetal face, demonstrating micrognathia.

A short femur is generally considered to be one measuring below the fifth percentile or below two standard deviations from the mean for gestational age. Short femurs most frequently are constitutional; however the wellrecognized association of this finding with fetal trisomy 21 must be considered. Fetuses whose long bones measure more than three standard deviations below the mean should be suspected of having a skeletal dysplasia, especially if the head circumference is greater than the 75th percentile. A femur-to-foot length ratio $<1$ is also suggestive of a dysplastic condition.

Limb shortening presents in a variety of patterns, which are categorized as follows:

- rhizomelia - shortening of the proximal segment of the limb (femur and/or humerus)

- mesomelia - shortening of the middle segment of the limb (radius, ulna, tibia, and/or fibula)

- acromelia - shortening of the distal segment of the limb (hands and feet)

- micromelia - shortening of all parts of the limb.

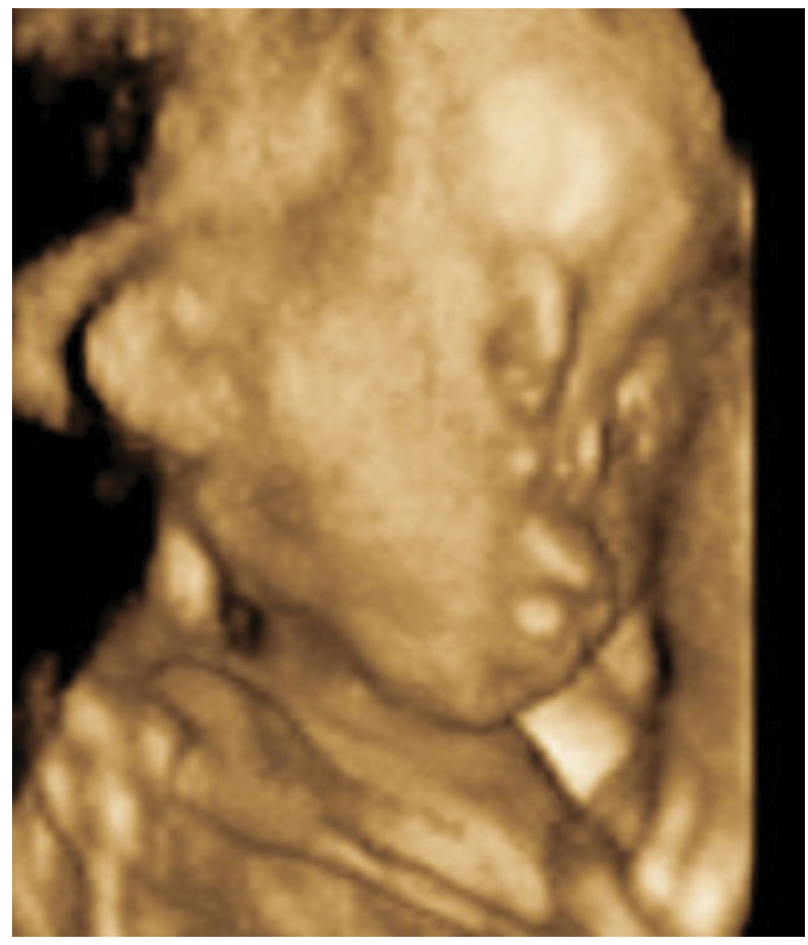

Figure 4 3-D rendering of the fetal face, showing mid-facial hypoplasia.

All of these can be further subdivided into mild, mild with bowing, or severe.

Evaluation of the long bones should also include analysis of the bone mineralization, inspection of the ossifications centers, documentation of any curvature, metaphyseal flaring (widening observed at the level of the metaphyseal plate), or metaphyseal cupping, although metaphyseal cupping and bone density are not always easy to evaluate by ultrasound. The presence of abnormal angulation of the bone shaft, of wrinkled or thick contours to the shaft, and of callous formation may all be suggestive of a fracture. Joint deformities, disruption, or incongruence are compatible with luxations or joint dislocations ${ }^{18}$ (Figure 1).

Detailed evaluation of the thorax is essential. The transverse thoracic diameter and chest circumference should be measured. Measurements that lie below the fifth percentile may indicate an increased risk for the development of pulmonary hypoplasia. The ribs also need to be assessed with respect to their size, shape, and the presence of fractures. Examination of the shoulder girdle (the clavicles and scapulae) can offer important clues to the diagnosis, eg, the scapulae are hypoplastic and rectangular in campomelic dysplasia, and both small scapulae and short ribs can be seen in thanatophoric dysplasia.

Assessment of the spine along its full length evaluates its regularity, potential abnormal curvatures, or incomplete closure of the neural tube. The vertebral body heights should 
Table 3 A systematic approach to ultrasound evaluation of the fetal skeleton

I. Measure all long bones

2. Compare with other segments, and classify the limb shortening as a) rhizomelia, b) mesomelia, c) acromelia, d) severe micromelia

3. Qualitative assessment of long bones

a) bowing, b) demineralization, c) fractures, d) metaphyseal flaring, e) absence of bones

4. Measure chest dimensions

a) thoracic diameters (anteroposterior and transverse), b) cardio/ thoracic ratio

5. Evaluate hands and feet

a) digits (polydactyly/syndactyly), b) positional deformities

6. Examine the form and structure of

Shoulder girdle - scapula, clavicle

Rib cage

Pelvic girdle

7. Evaluate the cranium

a) macrocrania, b) frontal bossing, c) cloverleaf skull, d) microcephaly

8. Evaluate the face

a) facial clefting, b) hypotelorism, c) hypertelorism, d) retro/ micrognathia

9. Examination of the spine

a) platyspondyly, b) demineralization, c) hemivertebrae, d) coronal clefts, e) vertebral disorganization

10. Evaluate internal organs, including echocardiography

II. Fetal motion

Overall movement, as well as movement and range of motion at specific joints (eg, associated also with atypical posturing of the extremities)

12. Amniotic fluid volume

be subjectively estimated and compared with adjacent disk height. Marked platyspondyly (where the vertebral body height is less than the disk height) can be diagnosed by ultrasound. The regularity of the ossification centers of the vertebra should be visualized in both sagittal and coronal planes.

The skull should always be carefully measured to exclude micro- or macrocephaly. The shape and mineralization of the skull bones can be very revealing. In cases where there is easy visualization of the proximal cerebral structures or cranial vault distortion upon probe pressure, poor bone mineralization should be suspected (eg, in osteogenesis imperfecta). Cloverleaf deformity of the skull or scapho/brachycephaly may suggest craniosynostosis.

Common facial abnormalities include clefting (lip and/or palate), retro- or micrognathia, midface hypoplasia, saddlenose deformity, and frontal bossing (as in achondroplasia). In the detailed evaluation of the fetal face and facial bones, the interorbital and extraorbital distances should always be measured to determine the presence of hypo/hypertelorism.

The bony pelvis and the presence of its three ossification points (iliac, pubic, and ischial bones) can provide valuable information. The shape of the iliac bone (round, flat, lack of iliac flaring) is important but often difficult to appreciate with 2-D ultrasound; similarly, depiction of the sacrosciatic notches is also difficult with conventional 2-D ultrasound.

Hands and feet should be carefully examined to exclude polydactyly (pre- and postaxial polydactyly should be differentiated), syndactyly, or clinodactyly. The hitchhiker's thumb is a rare finding, but suggests diastrophic dysplasia, while rocker-bottom or clubbed feet are often seen in aneuploidies, as well as a variety of conditions that may affect skeletal development, and form part of the differential diagnosis for skeletal dysplasias, including such conditions as multiple pterygium syndrome.

\section{Ultrasound evaluation of lethality}

Lethality is important to identify, as this will be key in counseling and also in defining the place for subsequent obstetric interventions (such as cesarean delivery) in ongoing pregnancies. The most manifest predictor of lethality is the finding of a hypoplastic thorax. ${ }^{18}$ Several methods have been described to assess thoracic hypoplasia. ${ }^{17}$ The key points are:

- chest biometry/features

o thoracic circumference below the fifth percentile, measured at the level of the four-chamber heart view

o thoracic-to-abdominal circumference ratio less than $0.6-0.79$

o short thoracic length (from the neck to the diaphragm, plotted on nomograms)

o ribs that encircle less than $70 \%$ of the thoracic circumference at the level of the four-chamber cardiac view

o markedly narrowed anteroposterior thoracic diameter in the sagittal view

o concave or bell-shaped contour of the thorax in the coronal view

o the ratio of (chest area - heart area)/chest area being below the fifth percentile

o chest:trunk-length ratio equal to or less than 0.32 .

- lung biometry

o lung area below the fifth percentile

o right lung diameter below the fifth percentile

o right lung area:thoracic area ratio below 0.11 .

- femur length/abdominal circumference ratio below 0.16

- Doppler evaluation of the distal pulmonary arteries

- Doppler assessment of tracheal fluid flow.

A comparative study of eight different methods for the prediction of fetal lung hypoplasia determined that the lung volumes and the thoracic circumference to abdominal circumference ratios performed best. However, the majority 
of these studies were performed in the context of fetal congenital diaphragmatic hernia, and these data may not extrapolate accurately to this population. ${ }^{29}$

The most reliable sonographic sign in the lethal skeletal dysplasias that are associated with decreased mineralization (such as osteogenesis imperfecta type II, achondrogenesis, or hypophosphatasia) is evidence of increased compressibility of the calvarium when pressure is applied with the ultrasound probe. ${ }^{30-32}$

In general, the earlier in the pregnancy that a skeletal dysplasia presents, the worse the prognosis. Therefore, the majority of cases found in the first trimester represent lethal skeletal dysplasias. ${ }^{10}$

\section{Computed tomography}

$\mathrm{CT}$ can be used in both 2-D and 3-D approaches. This modality should only be considered after ultrasound evaluation. Depending on the equipment used, the acquisition settings can be very different, but typically a scout view of the maternal abdomen is obtained to localize the fetus and to analyze its position. The portion of the skeleton to be visualized is then selected and examined using a maximum number of 30 scans, focusing where needed to minimize overall irradiation. ${ }^{33}$

CT allows fast and continuous acquisition of images, as the X-ray source and detector complete spiral or helical movements around the patient, who is moving in a linear fashion through the gantry. Helical CT allows fast and continuous acquisition of a volume data set containing the structures of interest, usually within one breath hold (20-25 seconds), thereby minimizing motion artifacts. Fast acquisition allows improved multiplanar reformatting and 3-D reconstruction. ${ }^{17}$

The main advantage of 3-D helical CT is the ability to visualize the entire fetus, and in the context of this subject it permits complete visualization of the fetal skeleton without the maternal bone superposition that occurs with conventional radiography. This specifically improves the visualization of the fetal vertebral column, the pelvic bones, and the various ossification centers. ${ }^{18}$ The 3 -D representation of a malformation that can be generated by CT may also be of value during prenatal parental counseling by allowing them to visualize the defect more easily. ${ }^{33}$

3-D helical CT has been previously used as an adjunctive diagnostic imaging modality for prenatal diagnosis of congenital anomalies in cases of trisomy 18 , cystic hygroma, congenital diaphragmatic hernia, and agnathia-holoprosencephaly. Long-bone measurements obtained by postmortem helical CT studies have been compared with those obtained within 24 hours of delivery by ultrasound, and a significant correlation between the two methods was observed. ${ }^{17}$ Consequently, CT imaging may be of value in the evaluation of fetuses where demise has occurred or prior to pregnancy termination where the parents will not allow for an autopsy examination.

There are several reported cases of skeletal dysplasias being diagnosed with $\mathrm{CT}^{34-37}$ Small studies have shown the considerable utility of $\mathrm{CT}$ as an adjunct to ultrasound, and demonstrated the diagnostic accuracy that can be achieved. Ruano et $\mathrm{al}^{23}$ demonstrated that 3-D CT was equivalent to 3-D ultrasound as a diagnostic tool. Table 4 summarizes the diagnostic accuracy of CT when used for the prenatal diagnosis of skeletal dysplasias. ${ }^{23,35,38}$

Recently, Macé et al concluded that from 26 weeks of gestation, helical CT had a sensitivity of $82 \%$, specificity of $91 \%$, and positive and negative predictive values of $90 \%$ and $83 \%$, respectively, for diagnosing fetal skeletal dysplasias. ${ }^{4}$ The prevalence of skeletal dysplasias was increased by including cases of micromelia (long bones below the third percentile) or if long bones were below the tenth percentile in length but in conjunction with other bony anomalies. In a retrospective study of 21 cases with fetal bony anomalies, Victoria et al demonstrated that $\mathrm{CT}$ revealed additional osseous findings not identified by ultrasound that in some cases influenced the perinatal management. They advocated the superiority of $\mathrm{CT}$, given that there were no cases in this series in which the CT findings differed incorrectly from the ultrasound findings and blinded review of the images revealed that CT outperformed ultrasound $(P<0.001){ }^{39}$

However, despite these promising results, $\mathrm{CT}$ has some limitations. To begin with, very few studies have evaluated the radiation safety of this technique in a large cohort, and given that there is inevitable radiation exposure that is potentially harmful, it remains contentious whether fetal CT can be justified under the ALARA (as low as reasonably achievable) principles of radiation protection. ${ }^{40}$ However, given that there are techniques that allow minimization of the radiation dose, this should not preclude the use of this technique where it is believed that the benefits outweigh the risks.

Table 4 Diagnostic accuracy of computed tomography for prenatal diagnosis of skeletal dysplasias

\begin{tabular}{|c|c|c|c|}
\hline Study & Year & $\mathbf{n}$ & $\%$ \\
\hline Macé et $\mathrm{al}^{4}$ & 2013 & 34 & $56(19)$ \\
\hline Miyazaki et al ${ }^{40}$ & 2012 & 17 & $100(17)$ \\
\hline Cassart et al ${ }^{38}$ & 2007 & 11 & $73(8)$ \\
\hline Ruano et $\mathrm{a}^{23}$ & 2004 & 6 & $100(6)$ \\
\hline
\end{tabular}


Another limitation is that CT does not allow for precise and complete visualization of the fetal extremities (in particular, the hands and feet), nor appreciation of bone mineralization. Furthermore, its value is of greatest benefit in the rarer and more complex conditions that are most typically diagnosed in the third trimester; therefore, the potential benefits of using $\mathrm{CT}$ to aid prenatal diagnosis at this late stage may be of more limited clinical value. ${ }^{18}$ The availability of skilled radiologists able to interpret fetal CT images accurately is also limited.

\section{Magnetic resonance imaging}

The more widespread availability of MRI and the fact that there is no radiation exposure have led to an increasing number of fetal indications for this technique being recognized. There are a number of case reports that have been published describing the use of fetal MRI in the evaluation of skeletal abnormalities. MRI potentially may offer some advantages over $\mathrm{CT}$ and ultrasound, in that it permits not only a large field of view but also accentuates soft-tissue contrast, and thus, unlike ultrasound, can be used to delineate abnormalities of fetal cartilage in detail. Additionally MRI, both in this context and in that of other fetal pathologies (such as congenital diaphragmatic hernia), has been used to evaluate fetal lung volume and thus provide some information regarding the risk of pulmonary hypoplasia. However, at present, the value of MRI in the detailed evaluation of fetal skeletal abnormalities remains unproven ${ }^{41}$

\section{Plain radiography}

Historically, radiographs of a fetus in utero in cases suspected to have a skeletal dysplasia were used to confirm the ultrasound findings and aid in counseling and planning case management. Although radiographs of the maternal abdomen may help visualize the fetal skeleton and identify possible abnormalities in bone shape and size, this is again most beneficial later in pregnancy, mainly after 30 weeks of gestation. ${ }^{18}$ It is our opinion that with the marked improvements in ultrasound imaging in the last two decades and given the poor resolution of skeletal radiographs obtained in utero, as well as a lack of expertise in the analysis of such radiographs, there is little value to this methodology in current practice. ${ }^{5}$

\section{Postmortem imaging}

Radiography, CT, and MRI are all techniques that may be of value in postmortem fetal or neonatal assessment. They can be useful in establishing correlations with antenatal ultrasound findings. In cases where conventional autopsy is declined, a virtual autopsy, with or without directed tissue biopsies, may be performed using either CT or MRI, with results that can approach the detail obtained by open autopsy. ${ }^{42,43}$

\section{Fetal skeletal abnormalities}

Even the commoner skeletal dysplasias occur in less than one in 10,000 births, while the rarer types occur in fewer than one in 100,000 births. The following section describes the ultrasound findings of some of the more often-identified skeletal dysplasias.

\section{Thanatophoric dysplasia}

This is the commonest lethal dysplasia, caused by de novo mutations in the FGFR3 gene. Prenatal ultrasound findings depend on the specific type (I or II). It is characterized by severe micromelia or rhizomelia, normal trunk length, a narrow thorax, brachydactyly, and platyspondyly. Head circumference is increased in $50 \%$ of cases with a depressed nasal bridge. Bone mineralization is normal and no bone fractures are seen in either type. In type I, the more frequent, there is also presence of long-bone bowing (characteristically described as resembling a telephone receiver), while craniosynostosis (cloverleaf skull) is more often a feature of type II. ${ }^{9}$ Holoprosencephaly, agenesis of the corpus callosum, and ventriculomegaly may also be associated with cloverleaf deformity, and there may be renal and cardiovascular anomalies. The skin appears thick because of extreme redundancy, and polyhydramnios is observed in approximately $50 \%$ of cases. ${ }^{44}$

\section{Achondroplasia}

Achondroplasia is the commonest nonlethal skeletal dysplasia that is also associated with mutations in the FGFR3 gene. Its features comprise micromelia, rhizomelia, mild limb bowing, brachydactyly, and particularly an increased interspace between the third and the fourth digits. It is characterized by an enlarged head circumference, with frontal bossing and mid-facial hypoplasia. However, not all these characteristics are necessarily present before the third trimester; therefore, at 18-22 weeks, fetal biometry may well be completely normal. ${ }^{44}$

\section{Osteogenesis imperfecta}

Bone fragility is the main characteristic of this group of conditions. There are many forms - eight are currently recognized - that vary in severity and consequently in the ultrasound findings observed. Type II is the most lethal form, and prenatal diagnosis of this variant has been described in 
several case series using both 2-D and 3-D ultrasound and CT. ${ }^{45}$ Type II is characterized by a compressible thin calvaria (which at sonography may be transparent), severe micromelia and bowing of the long bones, with multiple fractures (including the ribs) and a narrow thorax. ${ }^{44}$ The features of type III overlap with type II, although the latter tends to present earlier, and displays more marked shortening of the limbs, thinner and more fractured ribs, and a greater degree of translucency or undermineralization of the calvarium. Prenatal biochemical and genetic testing may be possible if the familial mutation has been identified or if collagen studies have been performed. ${ }^{46}$

\section{Achondrogenesis}

Two types of achondrogenesis exist, each having distinct histological and radiological features. Type I, accounting for $20 \%$ of cases, is a disorder of both endochondral and membranous ossification, characterized by a partial or complete lack of ossification of the calvaria and spine, as well as micromelia, and frequently multiple rib fractures. This type may be further subdivided (IA or IB) based upon genetic analysis. Type II accounts for $80 \%$ of cases, and is solely a disorder of endochondral ossification. It is less severe than type I, and involves a varying degree of calcification of the calvaria and spine. Fractured ribs are not present, and skull ossification is relatively normal. Prenatal ultrasonographic features include severe bone shortening with bowing, decreased mineralization, and a shortened trunk with a narrow, barrel-shaped thorax. Vertebral body ossification is lacking in both types, and is often more pronounced in the distal spine. The head is disproportionately enlarged, and severe fetal hydrops and polyhydramnios are often present. In type I, the skull, which is poorly ossified, allows for easy visualization of the intracranial contents, and is easily compressible with the transducer. The ribs are thin in type I, but generally thicker in type II. ${ }^{44}$

\section{Chondrodysplasia punctata}

Rhizomelic chondrodysplasia punctata is usually lethal within infancy or early childhood. There is a marked shortening and disrupted ossification of the proximal limb bones - rhizomelia - with associated abnormalities of the vertebral column and less marked femoral shortening. The epiphyses appear expanded, and contain multiple hyperechoic foci. ${ }^{44}$ Two forms of X-linked chondrodysplasia punctata are recognized, which can be associated with a normal life span.

\section{Campomelic dysplasia}

This disease, typically associated with anomalies in the vicinity of the $S O X 9$ gene, is characterized by prominent bowing of the long bones, especially those of the lower extremities. The fibulae may be hypoplastic or absent, and clubfoot or talipes is frequent. The scapulae are hypoplastic with a rectangular appearance or absent. There is relatively mild narrowing of the thorax. Facial features include a flattened nasal bridge, protuberant forehead, hypotelorism, an elongated philtrum, micrognathia, and a cleft palate. Associated cardiac anomalies are common, as are hydrocephalus, hydronephrosis, and polyhydramnios. ${ }^{44} \mathrm{~A}$ large proportion of affected fetuses may display genital ambiguity.

\section{Conclusion}

The spectrum of skeletal malformations that can affect the fetus or newborn is vast, with several hundred genetic conditions now being recognized. This breadth of pathology makes even postnatal diagnosis - when complete imaging, physical evaluation, and genetic testing can be done with impunity - challenging and often incomplete or inconclusive. Precise prenatal diagnosis is additionally hampered by the restrictions of accessibility to the fetus and the risks that imaging or invasive testing may carry.

As the number of patients with a family history of a skeletal dysplasia is small, it is the routine 2-D ultrasound assessment of fetal anatomy during pregnancy that remains key in the detection of suspected skeletal malformations. Improvements in ultrasound imaging technology, the use of other imaging techniques as complementary adjuncts to ultrasound, and our improved knowledge of the differential phenotypes and imaging characteristics of these varied conditions allow a working differential diagnosis to be established in pregnancies in which a fetal skeletal malformation is identified. Genetic analysis may allow such differentials to become focused on a specific condition or specific group of conditions. Our ability to discriminate between the lethal conditions or variants has also improved, which is key to parental counseling.

As these conditions are individually and even cumulatively very rare, it is important in examining a fetus with a suspected skeletal abnormality to adopt a systematic and thorough approach to the evaluation of the fetal skeleton, so that the phenotype can be described as precisely and completely as possible. This will give the fetal medicine specialists and prenatal geneticists the best opportunity to derive an accurate differential diagnosis, which will be used to guide the care for the remainder of the pregnancy and will also better enable neonatologists and other pediatric specialists, such as orthopedists, to discuss the postnatal ramifications of these conditions with the parents. It is important to emphasize 
that many of these conditions develop dynamically with advancing gestational age; therefore, it is also important to repeat such evaluations regularly to assess the evolution of the disorder. This too may help to refine the diagnoses.

Continuing advancements in our knowledge of the underlying genetic and biochemical origins of many of the skeletal malformations, as well as advances in genetic testing, will no doubt in the future contribute to more precise molecular genetic diagnoses. It is not far-fetched to imagine the future development of a gene array specifically constructed for the analysis of fetal and childhood skeletal malformations. However, even in the presence of such technologies, there will still be a place for the accurate recognition and description of skeletal anomalies to guide both parental counseling and diagnostic testing.

\section{Disclosure}

The authors report no conflicts of interest in this work.

\section{References}

1. Warman ML, Cormier-Daire V, Hall C, et al. Nosology and classification of genetic skeletal disorders: 2010 revision. Am J Med Genet A. 2011;155A(5):943-968.

2. Orioli IM, Castilla EE, Barbosa-Neto JG. The birth prevalence rates for the skeletal dysplasias. J Med Genet. 1986;23(4):328-332.

3. Weldner BM, Persson PH, Ivarsson SA. Prenatal diagnosis of dwarfism by ultrasound screening. Arch Dis Child. 1985;60(11):1070-1072.

4. Macé G, Sonigo P, Cormier-Daire V, et al. Three-dimensional helical computed tomography in prenatal diagnosis of fetal skeletal dysplasia. Ultrasound Obstet Gynecol. 2013;42(2):161-168.

5. Krakow D, Lachman RS, Rimoin DL. Guidelines for the prenatal diagnosis of fetal skeletal dysplasias. Genet Med. 2009;11(2):127-133.

6. Kurtz AB, Wapner RJ. Ultrasonographic diagnosis of second-trimester skeletal dysplasias: a prospective analysis in a high-risk population. J Ultrasound Med. 1983;2(3):99-106.

7. Parilla BV, Leeth EA, Kambich MP, Chilis P, MacGregor SN. Antenatal detection of skeletal dysplasias. J Ultrasound Med. 2003;22(3): 255-258.

8. Krakow D, Alanay Y, Rimoin LP, et al. Evaluation of prenatalonset osteochondrodysplasias by ultrasonography: a retrospective and prospective analysis. Am J Med Genet A. 2008;146A(15):1917-1924.

9. Schramm T, Gloning KP, Minderer S, et al. Prenatal sonographic diagnosis of skeletal dysplasias. Ultrasound Obstet Gynecol. 2009; 34(2):160-170.

10. Khalil A, Pajkrt E, Chitty LS. Early prenatal diagnosis of skeletal anomalies. Prenat Diagn. 2011;31(1):115-124.

11. Ngo C, Viot G, Aubry MC, et al. First-trimester ultrasound diagnosis of skeletal dysplasia associated with increased nuchal translucency thickness. Ultrasound Obstet Gynecol. 2007;30(2):221-226.

12. Dyson RL, Pretorius DH, Budorick NE, et al. Three-dimensional ultrasound in the evaluation of fetal anomalies. Ultrasound Obstet Gynecol. 2000;16(4):321-328.

13. Steiner H, Spitzer D, Weiss-Wichert PH, Graf AH, Staudach A. Threedimensional ultrasound in prenatal diagnosis of skeletal dysplasia. Prenat Diagn. 1995;15(4):373-377.

14. Leung KY, Ngai CS, Chan BC, Leung WC, Lee CP, Tang MH. Three-dimensional extended imaging: a new display modality for three-dimensional ultrasound examination. Ultrasound Obstet Gynecol. 2005;26(3):244-251.
15. Bega G, Lev-Toaff A, Kuhlman K, Kurtz A, Goldberg B, Wapner R. Three-dimensional ultrasonographic imaging in obstetrics: present and future applications. J Ultrasound Med. 2001;20(4):391-408.

16. Benacerraf BR, Shipp TD, Bromley B. Three-dimensional US of the fetus: volume imaging. Radiology. 2006;238(3):988-996.

17. Goncalves LF, Espinoza J, Mazor M, Romero R. Newer imaging modalities in the prenatal diagnosis of skeletal dysplasias. Ultrasound Obstet Gynecol. 2004;24(2):115-120.

18. Cassart M. Suspected fetal skeletal malformations or bone diseases: how to explore. Pediatr Radiol. 2010;40(6):1046-1051.

19. Hata T, Aoki S, Akiyama M, Yanagihara T, Miyazaki K. Threedimensional ultrasonographic assessment of fetal hands and feet. Ultrasound Obstet Gynecol. 1998;12(4):235-239.

20. Krakow D, Williams J 3rd, Poehl M, Rimoin DL, Platt LD. Use of three-dimensional ultrasound imaging in the diagnosis of prenatal-onset skeletal dysplasias. Ultrasound Obstet Gynecol. 2003;21(5):467-472.

21. Garjian KV, Pretorius DH, Budorick NE, Cantrell CJ, Johnson DD, Nelson TR. Fetal skeletal dysplasia: three-dimensional US - initial experience. Radiology. 2000;214(3):717-723.

22. Viora E, Sciarrone A, Bastonero S, Errante G, Botta G, Campogrande M. Three-dimensional ultrasound evaluation of short-rib polydactyly syndrome type II in the second trimester: a case report. Ultrasound Obstet Gynecol. 2002;19(1):88-91.

23. Ruano R, Molho M, Roume J, Ville Y. Prenatal diagnosis of fetal skeletal dysplasias by combining two-dimensional and three-dimensional ultrasound and intrauterine three-dimensional helical computer tomography. Ultrasound Obstet Gynecol. 2004;24(2):134-140.

24. Johnson DD, Pretorius DH, Riccabona M, Budorick NE, Nelson TR. Three-dimensional ultrasound of the fetal spine. Obstet Gynecol. 1997;89(3):434-438.

25. Lee W, Blanckaert K, Bronsteen RA, Huang R, Romero R. Fetal iliac angle measurements by three-dimensional sonography. Ultrasound Obstet Gynecol. 2001;18(2):150-154.

26. American Institute of Ultrasound in Medicine. AIUM Practice Guideline for the Performance of a Musculoskeletal Ultrasound Examination. Laurel (MD): AIUM; 2012.

27. American College of Obstetricians and Gynecologists. ACOG Practice Bulletin no 101: Ultrasonography in pregnancy. Obstet Gynecol. 2009;113(2 Pt 1):451-461.

28. Cargill Y, Morin L, Bly S, et al. Content of a complete routine second trimester obstetrical ultrasound examination and report. J Obstet Gynaecol Can. 2009;31(3):272-275, 276-280.

29. Yoshimura S, Masuzaki H, Gotoh H, Fukuda H, Ishimaru T. Ultrasonographic prediction of lethal pulmonary hypoplasia: comparison of eight different ultrasonographic parameters. Am J Obstet Gynecol. 1996;175(2):477-483.

30. Kahyaoglu SD, N. Clinical and ultrasonographic hints for prenatal diagnosis and management of the lethal skeletal dysplasias: a review of the current literature. Acta Medica. 2012;1:11-18.

31. Berge LN, Marton V, Tranebjaerg L, Kearney MS, Kiserud T, Oian P. Prenatal diagnosis of osteogenesis imperfecta. Acta Obstet Gynecol Scand. 1995;74(4):321-323.

32. Dhouib M, Guirat N. [Lethal osteogenesis imperfecta. Prenatal diagnosis]. Presse Med. 2004;33(10):658-660. French.

33. Brunelle F, Sonigo P, Simon I. Fetal CT. Childs Nerv Syst. 2003;19(7-8): 415-417.

34. Bonnefoy O, Delbosc JM, Maugey-Laulom B, Lacombe D, Gaye D, Diard F. Prenatal diagnosis of hypochondroplasia: three-dimensional multislice computed tomography findings and molecular analysis. Fetal Diagn Ther. 2006;21(1):18-21.

35. Miyazaki O, Nishimura G, Sago H, Watanabe N, Ebina S. Prenatal diagnosis of chondrodysplasia punctata tibia-metacarpal type using multidetector CT and three-dimensional reconstruction. Pediatr Radiol. 2007;37(11):1151-1154.

36. Sohda S, Hamada H, Oki A, Iwasaki M, Kubo T. Diagnosis of fetal anomalies by three-dimensional imaging using helical computed tomography. Prenat Diagn. 1997;17(7):670-674. 
37. Yamada T, Nishimura G, Nishida K, et al. Prenatal diagnosis of short-rib polydactyly syndrome type 3 (Verma-Naumoff type) by three-dimensional helical computed tomography. J Obstet Gynaecol Res. 2011;37(2):151-155.

38. Cassart M, Massez A, Cos T, et al. Contribution of three-dimensional computed tomography in the assessment of fetal skeletal dysplasia. Ultrasound Obstet Gynecol. 2007;29(5):537-543.

39. Victoria T, Epelman M, Coleman BG, et al. Low-dose fetal CT in the prenatal evaluation of skeletal dysplasias and other severe skeletal abnormalities. AJR Am J Roentgenol. 2013;200(5):989-1000.

40. Miyazaki O, Nishimura G, Sago H, Horiuchi T, Hayashi S, Kosaki R. Prenatal diagnosis of fetal skeletal dysplasia with 3D CT. Pediatr Radiol. 2012;42(7):842-852.

41. Victoria T, Epelman M, Bebbington M, et al. Low-dose fetal CT for evaluation of severe congenital skeletal anomalies: preliminary experience. Pediatr Radiol. 2012;42 Suppl 1:S142-S149.

42. Thayyil S, Chitty LS, Robertson NJ, Taylor AM, Sebire NJ. Minimally invasive fetal postmortem examination using magnetic resonance imaging and computerised tomography: current evidence and practical issues. Prenat Diagn. 2010;30(8):713-718.

43. Thayyil S, Sebire NJ, Chitty LS, et al. Post mortem magnetic resonance imaging in the fetus, infant and child: a comparative study with conventional autopsy (MaRIAS Protocol). BMC Pediatr. 2011;11:120.

44. Lee SH, Cho JY, Song MJ, et al. Fetal musculoskeletal malformations with a poor outcome: ultrasonographic, pathologic, and radiographic findings. Korean J Radiol. 2002;3(2):113-124.

45. Marion MJ, Gannon FH, Fallon MD, Mennuti MT, Lodato RF, Kaplan FS. Skeletal dysplasia in perinatal lethal osteogenesis imperfecta. A complex disorder of endochondral and intramembranous ossification. Clin Orthop Relat Res. 1993;(293):327-337.

46. Byers PH, Krakow D, Nunes ME, Pepin M. Genetic evaluation of suspected osteogenesis imperfecta (OI). Genet Med. 2006;8(6): 383-388.
47. Gaffney G, Manning N, Boyd PA, Rai V, Gould S, Chamberlain P. Prenatal sonographic diagnosis of skeletal dysplasias - a report of the diagnostic and prognostic accuracy in 35 cases. Prenat Diagn. 1998;18(4):357-362.

48. Tretter AE, Saunders RC, Meyers CM, et al. Antenatal diagnosis of lethal skeletal dysplasias. Am J Med Genet. 1998;75(5):518-522.

49. Hersh JH, Angle B, Pietrantoni M, et al. Predictive value of fetal ultrasonography in the diagnosis of a lethal skeletal dysplasia. South Med J. 1998;91(12):1137-1142.

50. Doray B, Favre R, Viville B, Langer B, Dreyfus M, Stoll C. Prenatal sonographic diagnosis of skeletal dysplasias. A report of 47 cases. Ann Genet. 2000;43(3-4):163-169.

51. Moeglin D, Benoit B. Three-dimensional sonographic aspects in the antenatal diagnosis of achondroplasia. Ultrasound Obstet Gynecol. 2001;18(1):81-83.

52. Clementschitsch G, Hasenöhrl G, Steiner H, Staudach A. [Early diagnosis of a fetal skeletal dysplasia associated with increased nuchal translucency with 2D and 3D ultrasound]. Ultraschall Med. 2003;24(5): 349-352. German.

53. Wong GY, Wong SF, Chan WP, Ng WF. Three-dimensional ultrasound findings of spondylocostal dysostosis in the second trimester of pregnancy. Ultrasound Obstet Gynecol. 2006;27(5):580-582.

54. Shih JC, Peng SS, Hsiao SM, et al. Three-dimensional ultrasound diagnosis of Larsen syndrome with further characterization of neurological sequelae. Ultrasound Obstet Gynecol. 2004;24(1):89-93.

55. Soto E, Richani K, Goncalves LF, et al. Three-dimensional ultrasound in the prenatal diagnosis of cleidocranial dysplasia associated with B-cell immunodeficiency. Ultrasound Obstet Gynecol. 2006;27(5): 574-579.

56. Esser T, Rogalla P, Bamberg C, Kalache KD. Application of the threedimensional maximum mode in prenatal diagnosis of Apert syndrome. Am J Obstet Gynecol. 2005;193(5):1743-1745.
International Journal of Women's Health

\section{Publish your work in this journal}

The International Journal of Women's Health is an international, peerreviewed open-access journal publishing original research, reports, editorials, reviews and commentaries on all aspects of women's healthcare including gynecology, obstetrics, and breast cancer. The manuscript management system is completely online and includes

\section{Dovepress}

a very quick and fair peer-review system, which is all easy to use. Visit http://www.dovepress.com/testimonials.php to read real quotes from published authors. 